\title{
A complete survey on blood pressure monitoring devices and applications.
}

\author{
Jeyanthi N*, Gokulnath BV, Thandeeswaran R
}

School of Information Technology and Engineering, VIT University, Vellore, India

\begin{abstract}
Every organ in the body needs oxygen to work actively. The oxygen is supplied when the blood passes through each part of the body by means of arteries. Factors like smoking, alcohol, stress, over salt in the diet may cause the artery wall to get narrower. As a result, the blood cannot pass through the vital organ, as a result, the organs in the body cannot work properly. So it may result in the heart failure, coronary artery disease, chronic kidney disease, vision loss, etc. In most of the cases of the hypertension, there won't be any symptoms or alert in the body. But it may result in serious health problem which may leads to loss of life also. That's why hypertension is also called as silent killer. Mostly older age people are affected by hypertension and many people in the rural area are not aware of it. So devices like blood pressure monitor help them in monitoring their blood pressure periodically. This will lead to longer and healthy life style. This paper provides the survey about different blood pressure monitor. This will be helpful for both rural and urban peoples.
\end{abstract}

Keywords: Arteries, Coronary artery disease, Silent killer, Hypertension, Blood pressure monitor.

Accepted on February 22, 2018

\section{Introduction}

Heart pumps the blood when the heart beats. The blood is circulated throughout the body to supply oxygen and energy to each part of the body. While pumping the blood force is exerted on the walls of artery and it requires energy or strength for the blood to move through arteries. The force that makes the blood to move is blood pressure. Systolic and diastolic are the two terms that is used to express the blood pressure. The maximum pressure that is exerted during one heartbeat is the systolic pressure. When the pressure is minimal between two heartbeats is the diastolic pressure. The blood pressure is measured in terms of ( $\mathrm{mmHg}$ ) that is millimetres of mercury.

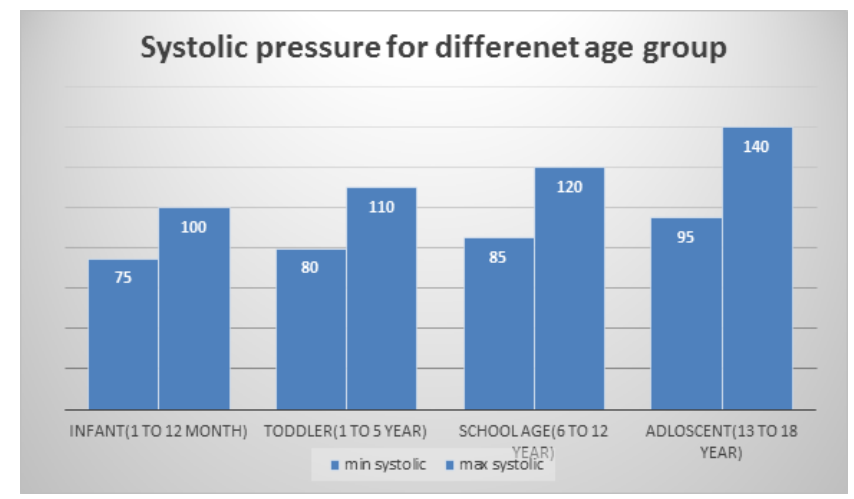

Figure 1. Minimum and maximum systolic pressure of different age groups.

Gold standard is the term used when the blood pressure is measured using mercury manometer and it gives the accurate reading as well. But the usage of mercury material is toxic. In the European countries, the usage of mercury manometer has been banned. Now a day's electronic, digital, wearable device are used to measure the blood pressure.

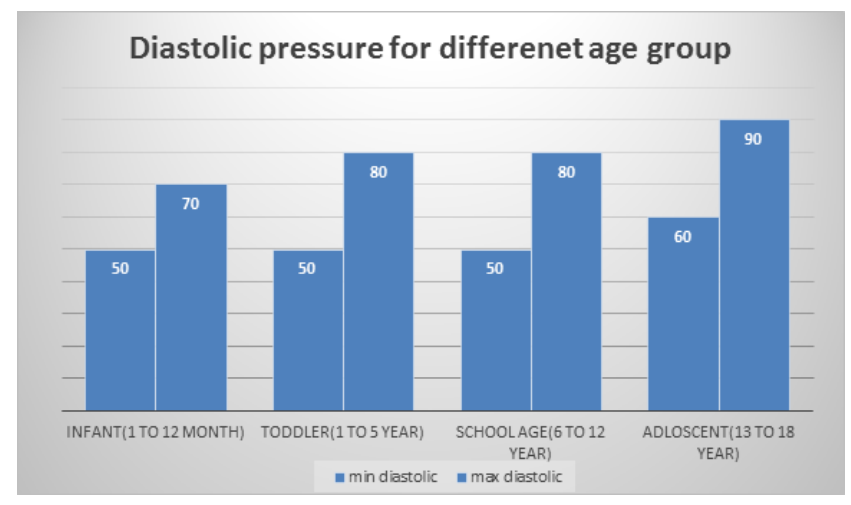

Figure 2. Minimum and maximum diastolic pressure of different age groups.

The effects and cause of the blood pressure leads to the discovery of blood pressure monitoring devices. Lack of activity physically, overweight, smoking, salt, alcohol consumption, stress, older age, genetic, kidney disease sleep apnea are some of the major causes for the hypertension. There are three stages in hyper tension and they are pre hypertension and it ranges from (120-139) over (80-90) and in the stage 1 it ranges from (140-159) over (90-99) and in the stage 2 it ranges from (160 and above) over (100 and above) (Figures 1-3). The high blood pressure has symptoms like nausea, vomiting, dizziness, diplopia, epistaxis, dyspnoea, palpitations. 


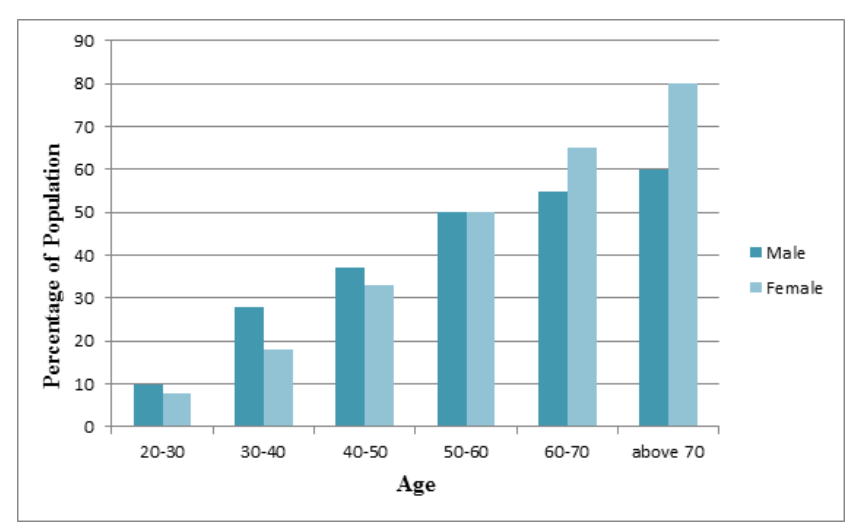

Figure 3. Percentage of male and female affected by hypertension.

\section{Measurement Location}

Brachial artery is standard part of the body to measure accurate blood pressure. Now a day's wrist and finger are also used to measure the blood pressure. But measuring the blood pressure at wrist and finger pressure gets varied greatly. When the blood passes through the body for each and every part it requires different pressure to move through the arteries. In the distal artery, the systolic pressure is high and diastolic pressure is low. Based on the emotional states like stress and happiness, health condition, cardiac output, arterial stiffness, total peripheral resistance and activity the blood pressure will vary. Right upper limb is the location of the brachial artery. The variation in the blood pressure for both right and left arm is small only.

Based on the position of the forearm also the pressure gets varied. When the arm is down the pressure is $(156-98 \mathrm{mmHg})$. When the arm position is horizontal then the pressure is $(139-84 \mathrm{mmHg})$. When the arm is up the pressure is (121-74 $\mathrm{mmHg})$.

The average of cardiac output, Systemic Vascular Resistance (SVR), and Central Venous Pressure (CVP) is the mean arterial pressure.

The average blood pressure in the artery for one cardiac cycle is the mean arterial pressure.

$M A P=(C O . S V R)+C V P$

Hence the CVP effort is small it is ignored

$M A P=C O . S V R$

MAP mainly helps in perfusion through different organ and it can be calculated using the systolic and diastolic pressure.

$M A P P_{\text {dias }}+1 / 3\left(P_{\text {sys }}-P_{\text {dias }}\right)$

The differences in the systolic and the diastolic pressure is the pulse pressure

$P_{\text {pulse }}=P$ sys $-P_{\text {dias }}$

\section{Common Blood Pressure Measurement Techniques}

For the standard measurement of blood pressure two techniques are used, they are auscultatory method and oscillometric method.

\section{Ausculatory method}

At first external pressure is given through cuff so that the arteries will become narrower and it doesn't allow the blood to pass through artery. Simultaneously by using stethoscope sound is noticed. As the arteries are narrowed there will be no sound and then gradually pressure is released through valve the point at which the arterial pressure exceeds the external cuff pressure a sound is heard. The sound is called as korotkoff sound.at the time of first sound heard readings are noted and it is the systolic pressure. Then slowly pressure is released through the valve and sound is heard continuously. When the blood pressure is greater than the external pressure there will be no sound and it is the diastolic pressure. The artery will be wider and the blood passes freely.

In auscultatory method also mercury is used. The mercury is banned due to its toxic nature; it brings a huge drawback in this method. So hybrid Sphygmomanometer is used it replace the mercury by combining both electronic pressure gauge and auscultatory device. And the measurement of blood pressure is calculated as auscultatory method.

\section{Oscillometric method}

It uses an electric pressure sensor for calculating the blood pressure. The cuff is inflated by automatically so that the arteries becomes narrow and it does not allow blood to pass through the artery and the pressure is released slowly it allow blood to pass through slowly and there will be vibration against the wall of artery and there will be cyclic expansion and contraction in the artery. And the point it reaches the highest cyclic value is noted and with the help of generic algorithm the systolic and diastolic pressure is calculated. The algorithms for each device vary according to the device used. There is no standard algorithm in it.

\section{Survey on Devices Used for Measuring Blood Pressure}

There are many electronic devices used for measuring the blood pressure. Some of the devices are wearable devices, some can be synchronized with mobile phones, some devices are fully automatic and some are semi-automatic devices. This survey gives a detail description about the features of the device, cost of the devices, memory capacity of the device, accuracy factor of the device, device portability, and technique used in the device and added features in the device (Figure 4). 


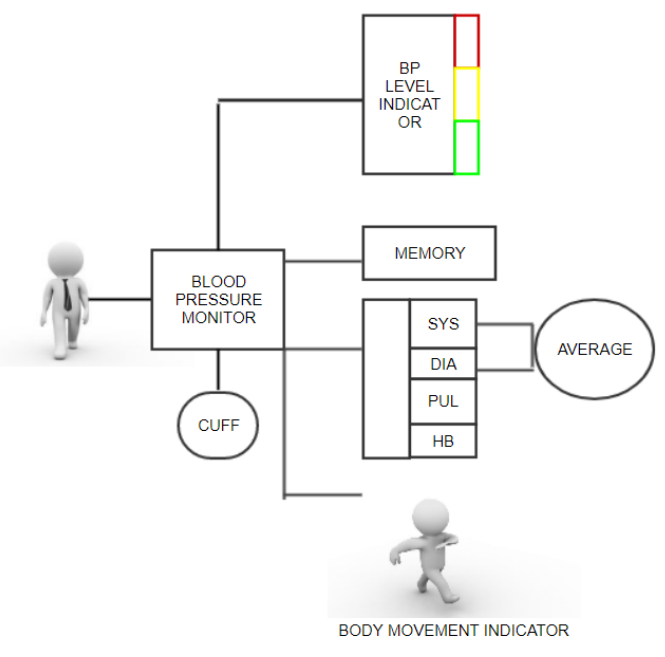

Figure 4. Functionality of blood pressure monitor device (Greennormal blood pressure, yellow-moderate blood pressure, red-high blood pressure).

\section{Omron blood pressure monitor device}

Omron HEM-7113 automatic blood pressure monitor [1] is a device used for indicating irregular blood pressure and hypertension and the accuracy pressure of the device is $\hat{\mathrm{A}} \pm 3$ $\mathrm{mmHg}$ and the percentage of display reading of pulse is $\hat{\mathrm{A}} \pm 5$. Its memory capacity allows 14 sets of reading. The components of Omron are main unit, medium cuff, battery set and instruction manual. It has warranty period of 1 year for the device. It cost around Rs1500/- and it's available in online shopping. It is used for the domestic purpose as well. It is mainly used for the patient who is suffering from chronic disease. Anxiety and stress related problems can also be found. The features of Omron are LCD digital display, oscillometric method and the pressure range is from $0 \mathrm{mmHg}$ to $299 \mathrm{mmHg}$ and for pulse 40 to $180 / \mathrm{min}$. The accuracy factor for pressure is $\pm 3 \mathrm{mmHg}$ and for the pulse is $\pm 5 \%$. The inflation is done by the electric pulse and deflation is by releasing the valve automatically. 4 AAA batteries are used as the power source.

Omron HEM-6131 automatic wrist blood pressure monitor device [2] has memory capability for 60 sets. It helps in body movement detection and finding the average value of last three irregular heartbeats. Detection of BP and irregular heartbeat are the key feature of this device. It has warranty period for one year. This device has patented Intelli sense technology for the purpose of comfort, fast and accurate reading. It is a portable device as well. The measurement posture guide in it brings you the accuracy in the measurement. The intuitive indicators present in it tell the optimum blood pressure value. The device had satisfied the accuracy factor provided by the European Society of Hypertension and Advancement of Medical Instrumentation. Its pressure ranges from 0 to 299 $\mathrm{mmHg}$ and pulse ranges from 40 to 180 beats $/ \mathrm{min}$. The accuracy for pressure is $\pm 3 \mathrm{mmHg}$ and for the pulse is $\pm 5 \%$ from display reading. The cost of the product is around Rs3300/-
Omron HEM-7117 JPN2 Blood pressure monitor device [3] has hypertension indicator in it. It helps in the detection of irregular heartbeat. It has separate wrapping cuff guide in it. Its mode of operation is one touch and the technology used is Intelli sense. It has a warranty period of 1 year. One button will bring quick result and detect irregular heartbeat. The Intelli sense technology enables re-inflation and pre-setting. There is an indicator present in the cuff wrapping which ensures cuff is wrapped at right position or not. 30 sets of reading can be recorded. When the blood pressure range falls under hypertension the heartbeat symbol starts to blink. The pressure value ranges from $0-299 \mathrm{mmHg}$ and pulse value ranges from 40-180 beats/min. the accuracy factor for pressure is \pm 3 $\mathrm{mmHg}$ and for the pulse is $\pm 5 \%$. The inflation process is controlled by electric pump and deflation process is by releasing valve. It cost around Rs2000/-.

Omron HEM-7120 automatic blood pressure monitor device [4] operation is one touch and it helps in the detection of the body movement and hypertension. It also helps in the irregular heartbeat detection. It stores the value of the last reading only. It display systolic pressure, diastolic pressure and pulse rate. Its manufacturer warranty is for one year. It's a fully automatic device and working principle of it is oscillometric method. The Intelli sense technology used in it helps in the inflation process. In case of hypertension, the heart symbol in it blinks. It cost around Rs1500/-.

Omron HEM 8712 blood pressure monitor device [5] helps in tracking the blood pressure which manages the health effectively. It mostly used by the clinical professionals. The features are body movement detection, blood monitor. It will save only the last reading. Irregular heartbeat detection is also one of the features of this device. It cost around Rs1500/-. This device is not recommended for child.

Omron HEM-7130-L blood pressure monitor [6] has level indicator for the blood pressure and it can store up to 60 memories of data. Each data will be displayed along with time and date. It also calculates the average value of the last three data. It features include irregular heart beat detection, body movement detection. It has warranty period of 5 years. The cuff that is provided is for the upper arm. It cost around Rs2600/-.

Omron HEM-7132 blood pressure monitor device [7] has intelligence technology in it. The memory capacity of the device is up to 60 measurement of reading. It is a light weighted device. The mode of operation is one touch. The features of it include body movement and finding irregular heartbeat. The components of the device are main unit, arm cuff, battery, and instruction manual. Five years of warranty is provided. It has equipped with body motion sensor. Its accuracy is $99.9 \%$. Inflation and deflation are provided by Intelli sense technology. It cost around Rs2600/-.

Omron HEM 8712 blood pressure monitor device [8] features are tracking blood pressure, managing health. It is mainly used by many clinical health professionals. It has special features like body movement detection and irregular heartbeat 
detection. The warranty period of the product is for one year. The components of it are larger display, battery. It's a super utility device. It cost around Rs1500/-.

Omron HEM-7121 blood pressure monitor [9] has features like hypertension indicator, body movement detection, and memory capacity of 30 readings, irregular heartbeat detection, and display systolic, diastolic pressure and pulse rate. It has warranty period of one year and it is extendable one. It has body motion tracking sensor in it. The reading of the blood pressure can be converted into hard copy using chip reader. It needs 4 AA batteries. This version of Omron helps in finding white coat hypertension, masked hypertension and morning hypertension. It has warranty period of 1 year and it cost around Rs1800/-.

Omron HEM-7112 blood pressure monitor device [10] ha pressure accuracy of $\pm 3 \mathrm{mmHg}$, pulse accuracy is \pm 5 percentage. The component of the device are main unit, medium size cuff, storage case with instruction manual and battery set and has 1 year of warranty as well. It helps in monitoring blood pre4ssure, irregular heartbeat. It has an error proof pressure up to $3 \mathrm{mmHg}$. Memory capacity stores 20 sessions value of blood pressure. It cost around Rs1400/-. Inflation and the deflation process is done automatically.

Omron HEM-7200 JPN1 blood pressure monitor [11] has blood level display in it and also detects irregular heartbeat. It works on oscillometric method. The accuracy factor is high in it. It stores up to 60 sets of readings. It calculates the average value of last three reading. The warranty period is for one year. The irregular heartbeat algorithm tells the result value is correct or not and whether it has to be calculated again or not. The pressure range is from $0-299 \mathrm{mmHg}$ and for the pulse is $40-180$ beats/min. The accuracy of the pressure is $\pm 3 \mathrm{mmHg}$ and for the pulse is $\pm 5 \%$. For the inflation purpose, fuzzy logic is used and the deflation process is automatic release valve. It cost around Rs3000/-.

Omron HEM 7270 blood pressure monitor device [12] has indicator for hypertension, blood pressure level, and irregular heartbeat. The component of the device are cuff guide, large cuff, display screen. The mode of operation is one touch and has guest mode option in it. The technology used is Intelli sense. It has warranty period of 1 year. It has memory capacity up to 60 sets of reading. The cuff diameter is of 22 to $42 \mathrm{~cm}$. It cost around Rs3600/-.

Omron HEM-6121 automatic wrist blood pressure monitor device [13] has Intelli sense technology in it. It features are irregular heartbeat detection, BP level indicator. 30 sets of reading value can be stored. The warranty period is for 1 year. The stored values are displayed along with date and time. The device is like wrist watch. It is tied over the wrist region. It cost around Rs2900/-.

Omron HEM-7117 JPN2 blood pressure monitor [14] features are hypertension indicator, irregular heartbeat indicator. It has one touch operation and Intelli sense technology. The warranty period is of 1 year. Its memory capacity is of 30 readings. The hypertension indicator is built in. The method used for the measurement is oscillometric method. The pressure range is from 0-299 $\mathrm{mmHg}$ and for pulse $40-180$ beats $/ \mathrm{min}$. The components of the device are main unit, medium or large size cuff, instruction manual, case for storage and battery. The battery life is up to 300 measurements. It cost around Rs2100/-.

Omron 10 series wireless upper arm blood pressure monitor device [15] is recommended by many pharmacists. It measure reading up to 200 readings. It is synchronized with smartphones like I phone and android phones. Three times readings are taken consecutively with $1 \mathrm{~min}$ apart and average of those reading are calculated. It has comfit cuff that has to be tied around the upper arm. It has two sizes in it they are medium and large. It has features like backlight, large screen, indicator light, quick start guide, and AC adapter. It has blue tooth connection in it. The green colour light represents the normal level of BP and orange colour indicates hypertension. There is bar level display for BP that compares the current BP reading to normal BP levels. It cost around Rs8000/-.

Omron HEM-7200 (JPN-1) blood pressure monitor device [16] display blood pressure level and the method used is oscillometric method. It helps in body movement detection, irregular heartbeat detection and the memory capacity is up to 60 reading of measurement. The time taken for calculating the average of last 3 reading is $10 \mathrm{~min}$. It has the warranty period for 1 year. It cost around Rs2900/-.

Omron HEM-7124 blood pressure monitor [17] has Intelli sense technology in it and the mode of operation is one touch and it has larger screen of display and stores the value of BP up to last reading (Table 1). The hypertension indicator is present in the device. It will give alert when the blood pressure falls under hypertension range. The device is simple and accurate. It cost around Rs1700/-.

Table 1. Advanced feature of Omron 7 series.

\begin{tabular}{|c|c|c|c|c|c|c|}
\hline & BP742N & BP761 & BP760N & BP785N & BP786 & BP710N \\
\hline BP colour level indicator & No & Yes & Yes & Yes & Yes & No \\
\hline Two user mode & Yes & Yes & Yes & Yes & Yes & No \\
\hline Memory storage & 100 & 120 & 120 & 200 & 200 & 14 \\
\hline
\end{tabular}




\begin{tabular}{|c|c|c|c|c|c|c|}
\hline Bluetooth & No & Yes & No & No & yes & No \\
\hline Accuracy & Yes & Yes & Yes & Yes & Yes & Yes \\
\hline BP level bar & Yes & Yes & Yes & Yes & Yes & No \\
\hline Average calculation & Yes & Yes & Yes & Yes & Yes & No \\
\hline Irregular heartbeat detection & Yes & Yes & Yes & Yes & Yes & No \\
\hline Battery & $4 \mathrm{AA}$ & $4 \mathrm{AA}$ & 4AA & AC adopter & AC adopter & $4 \mathrm{AA}$ \\
\hline Cuff type & D-ring & Easy-wrap & Easy-wrap & Easy-wrap & Easy-wrap & D-ring \\
\hline Cuff size in inches & 9 to 17 & 9 to 17 & 9 to 17 & 9 to 17 & 9 to 17 & 9 to 17 \\
\hline
\end{tabular}

\section{Equinox blood pressure monitor device}

Equinox digital blood pressure monitor EQ-BP-100 device [18] has features like lightweight, large display, portable, compact device. For the better accuracy purpose, DPDA technology is used. The inflation technique used is more comfortable for the users. It has one button control in it. The warranty period is for 18 months. For the defect and complain purpose separate customer care service is provided. It's a handy device with large LCD. For the battery purpose, 4 AAA battery is used. It can be used by the patient itself without getting help from others. It cost around Rs1000/-.

Equinox digital blood pressure monitor EQ-BP-101 device [19] helps in BP classification and irregular heartbeat detection. The components are large cuff, monitor, 4 AAA battery, Storage kit and the technology used is DPDA technology for providing the accuracy factor. The inflation technology is more comfort the users. It allow 60 measurement saving. The mode of power can be provided by either battery or adopter. Hand movement detection is special feature present in it. Manual instruction has to be strictly followed in-order to obtain the accurate value. It has 18 month of warranty. It cost around Rs1300/-.

Equinox digital blood pressure monitor EQ-BP-102 device [20] has larger LCD display, irregular heartbeat detection with blood pressure classification indicator, larger cuff size, 4 AAA batteries. The accuracy for the pulse is \pm 5 . There are two options available in the device battery and adapter. The BP power adopter monitor is provided for free. The accuracy factor will get varied if the instructions are not followed properly. The warranty period is for 18 months. For the service and the issue, user-friendly customer care is provided. The battery has to be removed while using the adopter. It cost around Rs2000/-.

Equinox EQ-BP 201 Palm type blood pressure monitor device [21] has ABS plastic, nylon cuff with D-ring, 1-tube PVC bladder, flat bottom with PVC bulb, nylon carrying bag, plastic end valve. It has $64.5 \mathrm{~mm}$ dials and manufacturer warranty for one year. The accuracy factor gets varied from other devices and it is low in it. The release valve is absent in it so that the cuff cannot be completely evacuated. The accuracy is varied mainly because of the air present in the cuff. It cost around Rs1800/-.
Equinox EQ-BP 54 upper arm blood pressure monitor device [22] measures the blood pressure automatically and there is an auto shut off function in it. The range of pressure is 20-280 $\mathrm{mmHg}$ and for the pulse is $40-199$ per min. The method used for measuring is oscillometric method. The components of the devices are main unit, 4 AA batteries, arm cuff, instruction manual and warranty card. The warranty period is for 1 year. The cost of the device is Rs1300/-.

Equinox Qbp digital blood pressure monitor EQ-BP-i104 device [23] features are large LCD display, Bluetooth, large size cuff. It also detects irregular heartbeat and the DPDA technology present in it mainly ensures the accuracy. The inflation technique is comfortable and deflation is an automatic process. There is a separate instruction manual given for it in order to obtain the accuracy. The special feature of this device is that it is synchronized with iFittr app and this app helps in maintaining the record. For the issue or defect, a user-friendly customer care is provided for it. This version of Equinox also has DPDA technology in it. It cost around Rs3000S/-.

\section{Health sense blood pressure monitor device}

Health sense BP100 Heart mate classic fully automatic digital talking blood pressure monitor [24] device has features like talking function, body movement indicator, blood pressure level voice command, $2 \times 90$ memory, find irregular heartbeat, display systolic pressure, diastolic pressure, pulse rate, alarm, larger LCD, wide cuff, low battery indicator and calculate average (Figure 5). There is a separate guideline for measurement, no not attempt factors, right posture. The medium of speech for talking function is English. It also has latest oscillometric method. It has the capacity to store 90 members measurement. The talking function in this device operates both at normal and noisy environment.

Health sense classic BP120 heart mate fully automatic digital blood pressure monitor device [25] has oscillometric technique, extra-large bright LCD, black font display, universal port (6 V USB devices). It mainly helps in monitoring hypertension. Hypertension is the main cause of heart problem, renal failure, and stroke. It helps in the detection of irregular heartbeat, body movement. It has memory capacity of 120 reading. The cuff arm size is $22-42 \mathrm{~cm}$ so it is comfortable for the user. It can be connected with devices like laptop, mobile charger, desktop, power bank. When the battery is low or there 
is an error in BP it will indicate us. The techno centric feature in it enables the response in one minute. It support USB device which helps in the multiple device connection. It also has WHO indicator in it. The WHO indicator present in it has six levels in it they are optimal, normal, G1-G3 and hype. It displays pressure value along with time and date. It has silicon feet for grip. It cost around Rs1300/-.

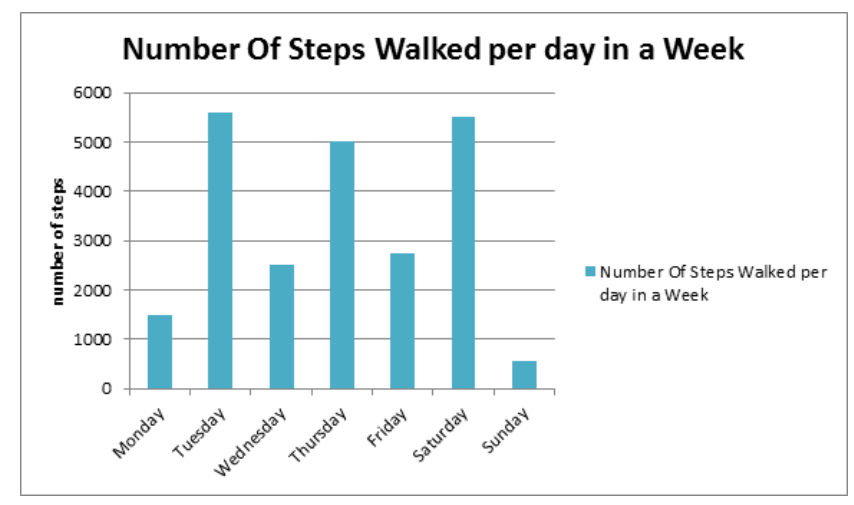

Figure 5. Body movement indicator graph.

Health sense BP300 heart mate digital upper arm blood pressure monitor device [26] has third generation MDI technology, automatic and oscillometric technique. It has large LCD screen. The memory capacity of it is it can store 2 user details and 60 reading. It main features are irregular heart beat detection, low battery, WHO indicator, larger cuff size. The time taken for calculating the average of 3 reading is 10 minutes. It cost around Rs2300/-.

\section{Dr trust blood pressure monitor device}

Dr Trust blood pressure monitor sliver line [27] is a device used to find the blood pressure. Its memory capacity allows 120 reading and 4 members value can be recorded. It has WHO indicator for indicating the level of blood pressure with classification. In case of low battery, it will indicate with beep sound. It uses oscillometric method for finding blood pressure. Automatic on and off, date display, irregular heartbeat, large LCD, wide soft cuff, calculates average is the features of it. Separate measurement guidance and right posture instruction are mentioned clearly. The inflation and the deflation process are done automatically. The pulse measurement range is 180 beat $/ \mathrm{min}$. The pressure accuracy is about $3 \mathrm{mmHg}$ and for the pulse is \pm 5 . Its price is around Rs1200/-.

Dr Trust (USA) Gold line fully automatic talking blood pressure monitor [28] with 3 colour hypertension backlight device has features like talking facility. This feature helps visually challenged person to know their health condition and there are 3 backlit indicators in it. Green colour shows normal blood pressure level. Yellow colour indicates pre hypertension. Red colour indicates hypertension. It has WHO indicator in it which is used as the standard for measuring blood pressure. The fuzzy algorithm present in it helps to eliminate over pumping. The cost of the device is Rs1800/-.
Bestest BP talk fully automatic digital talking blood pressure monitor device [29] uses most advanced fuzzy algorithm for maintaining high accuracy and it also avoids over pumping. The taking feature present in it is unique and makes easily understandable one. It has WHO indicator in it, which is the standard for calculation whether the blood pressure is high or low. There is bar indicator in it which has three colours namely green, yellow, and red. The green colour indicates the blood pressure is low and the yellow colour indicates the blood pressure is low and the red colour indicates the blood pressure is high. It has warranty period of one year. It has separate manual description for how to get accurate measurement and guideline for how to use the device. It cost around Rs1200/-.

\section{Dr Morepen blood pressure monitor device}

Dr. Morepen BP one BP3BG1 Blood Pressure Monitor device [30] has oscillometric measuring with both inflation and deflation is done automatically and it has fuzzy logic in it. It has features like high accuracy; battery capacity is good, one touch operation, large display screen and warranty for one year. It's an ambulatory monitoring device mainly used when there is fluctuating blood levels for the patients. It is used for both hospital and domestic purpose. It is mainly recommended for hypertension cases. It has battery indicator, high accuracy and memory back up. The disadvantage of it is store only last measured data and the cuff is not placing perfectly on arm. It cost around Rs1600/-.

Dr Morepen BP one BP09 fully automatic blood pressure monitor [31] has features like four interfaces. It involves in the detection of heartbeat and low battery. It has WHO blood pressure classification in it. It is user-friendly. It cost around Rs1200/-.

Dr Morepen 3.7 V BP-02UA blood pressure monitor device [32] mainly used for health monitoring and blood pressure measurement. It has warranty period of 1 year. The device is made up of plastic material. The power mode is battery and it is an inbuilt feature in it. For safety purpose, the device should be kept away from the children. It cost around Rs2300/-.

Dr Morepen SPG03 Aneroid blood pressure monitor device [33] is a digital blood pressure monitor with high quality. It doesn't have mercury in the meter. The components of the device are inflatable cuff, circular dial. It has special feature which will eliminate the calibration error. It is helpful in observing the cardiac cycle. The technique used in it is auscultatory method. It cost around RS750/-.

\section{Blood pressure monitor device}

Rossmax GB102 Aneroid blood pressure monitor device [34] components are chrome plated brass air release valve, latex free cuff, light weight stethoscope, black enamel $300 \mathrm{mmHg}$ non-stop pin reliable manometer. It is a digital sphygmomanometer which is patented. During drop also the calibration level is maintained. The design has spill proof in it. Backlight facility is also provided in it. It needs 2 AAA sized battery for the power sources which is capable for 3000 
measurements. The alert feature enables it to calibrate up to 10000 measurements. It cost around Rs1400/-.

Rossmax GB101 Aneroid blood pressure monitor [35] varies from GB101 by battery. GB101 version does not require battery sources. The components are chrome plated brass air release valve, latex free cuff, light weight stethoscope, black enamel $300 \mathrm{mmHg}$ non-stop pin reliable manometer.

Diamond LED Delux blood pressure monitor device [36] has rising spot LED present in it which indicates the pressure value. The functionality of mercurial instrument and diamond deluxe are similar. There are two mode of power operation in it they are battery and adopter. The advantage of it is low power consumption and easy to handle. There is special control valve for the deluxe cuff in it. The components are LED monitor, LED panel, USB port. The principle of this is similar to conventional sphygmomanometer. The pressure rise is indicated by rise in the mercury level. There is a dual control valve in the cuff for maintaining the accuracy in blood pressure. The mode of power is through battery or USB port. In rough condition also the device is reliable and accurate. The price of the device is around Rs2700/-.

37 degree Bluetooth smart bracelet is a wearable device [37] used as a fitness tracker and for measuring heart rate, blood pressure, fatigue, emotional status. It is synchronized with the smart phones like Samsung, HTC, Huawei, Xiao MI and iphone. During the measurement of heart beat, it also measure the number of calories burn, provide exercise goal. The step motion meter will help us to know about the movement and distance of walk which helps in the improvement of the health. The bracelet will automatically identify the sleep and wake up monitoring and also categorises the sleep into shallow sleep and deep sleep. Along with blood pressure detection, it helps in mood detection and helps to stay in good condition. As per the customer review, the product doesn't perform well. It costs around Rs2900/-.

JSB DBPO5 arm blood pressure monitor device [38] has WHO indicator for blood pressure classification. It will calculate the average value for the latest 3 measurement to ensure the reading is correct or not. The memory function unit of the device is $2 * 90$ groups. The components of the device are BP cuff, BP monitor, 4AA battery, carry case and instruction manual. The manufacturer warranty is for 1 year. Its type is oscillometric and also helps in finding the irregular heartbeat. It cost around Rs1000/-.

JSB DBP04 wrist blood pressure device [39] has WHO indicator in it. It will calculate average of the last three measurements. The memory capacity of the device is $2 \times 90$. The components of the device are wrist cuff, 2AAA battery, 1 manual, 1 carry case. The power to the device is supplied by 4 AA size battery. It has warranty period of 1 year. It cost around Rs1000/-.

Citizen CH-650 blood pressure monitor device [40] detect irregular heart beat and body movement. This helps in monitoring the health condition. It has the memory capacity of 60 reading and it calculates the average of last 3 readings. It has automatic power off function in it. Separate storage case is provided for it. It cost around Rs1800/-.

MCP digital blood pressure monitor BP110 device [41] has some special features like port for USB charging. It helps in detecting heart beat and pulse. As per the customer review the device not working properly and not giving accurate value. The memory capacity of it is $1 \times 199$ sets. It has two user features, larger cuff and it can store up to 500 readings. It cost around Rs1000/-.

MCP palm type blood pressure monitor device [42] use aneroid method for measuring the blood pressure. It is approved by Food and Drug Administration with pressure accuracy of $3 \mathrm{mmHg}$. The aneroid gauge is easy to use and produce accurate result. The components of the device are arm cuff, deluxe air release valve, inflation bulb, carrying case. It cost around RS1100/-.

INFI INF1112A 3rd generation digital BP monitor device [43] has third generation blood pressure monitor and larger cuff. The cuff is tied around the upper arm. This device gives accurate and faster reading. After the inflation process, it gives diastolic pressure value. Multiple measurements can be taken for comparing the least value. It helps in detecting the irregular pulse and WHO indicator in it. It has memory capacity up to 60 readings. It cost around Rs1500/-.

INFI Futura TMB-995 device [44] has same features like INFI INF $1112 \mathrm{~A}$ and it get varied in memory storage purpose. It allows storing 120 readings. Along with the device, free adopter is provided. It will check only inflation for calculating. If we measure diastolic first then it will result in measuring accurate diastolic value. Free adopter is provides along with the kit. It cost around Rs1500/-.

Citizen CH-453 Blood pressure monitor device [45] helps in the detection of irregular heartbeat and body movement. It has memory capacity up to 90 readings and also helps in calculating average of last three readings. It has features like automatic off, larger LCD and also has manufacturer warranty for 1 year. It cost around Rs1800/-.

Smart care mercury monitor device [46] has standard cuff in it and there is no D ring in it, standard latex bulb, and plastic connector. The mode of operation is hassle free. The cuff is made of nylon and the pin in the manometer is non-stop. This device can be used for both genders. It cost around RS650/-.

Newnik SP501 Sphygmomanometer device [47] uses aneroid method for measuring the blood pressure along with the device free stethoscope is provided. The component of it includes stethoscope, cuff, and storage kit. The zero position in the meter is easily adjustable one. It has non-stop pin in it. The measurement is quick and accurate. The pin is of $300 \mathrm{mmHg}$ in manometer. The air release valve is made up of chrome plate. It is certified by ISO and CE. The warranty of the product is for one year. It is also approved by FDA. It is latex free.

Smart care blood pressure dial type device [48] is mainly used for measuring the blood pressure. The component of the device are nylon cuff, there is no D ring in it, non-stop pin manometer, 
two tube with bladder in it, latex bulb, vinyl zipper bag, release valve with spring. The technique used in it for measuring the blood pressure is aneroid. It cost around RS700/-.

Withings wireless blood pressure monitors device [49] measures high accurate blood pressure value. It has automatic synchronization with mobile application and it is wireless. The resultant value and the complete description are done by health mate application. It also has wired and Bluetooth option in it. It can be used in both Android and iOS. It cost around RS33000/-. The feedback of the measurement is based on National Health Standards. It is verified by Food and Drug Administration. It is compatible with I phones, I watches.

I Health BP7 wireless device [50] used for measuring blood pressure, pulse wave, heart rate, and time. It maintain a chart to compare the current state of BP with past BP average and WHO classification. The results that are obtained can be shared with doctor and family and it also keep track of diet. It has got approved from Food and Drug Administration (FDA). Automatic wrist detection helps in predicting accurate blood pressure. It is synchronised with I Health application. Triple check feature helps in maintaining the accuracy factor. It also support for multiple users.

Cooey Smart Bluetooth and Voice based BP Meter [51] has features like Bluetooth, voice broadcast, rechargeable battery, tubeless. It can be synchronized with Android and iOS applications. It helps in measuring systolic, diastolic, irregular heartbeat. It is easy to carry. The technology used in it is (MWI) and it has MEMS sensors. The pressure accuracy in it is $\pm 3 \mathrm{mmHg}$ and pulse is $\pm 5 \%$. 4.0.4 Blue tooth precision sensor can also be added in it. $M$ health application in mobile phones helps in tracking the history. The progress of the patient can be tracked and shared at regular interval. The data's are stored in the cloud platform. IT cost around Rs2750/-.

Health genie Digital Upper Arm Talking blood pressure monitor device [52] has special features like talking ability. It mainly helps the blind and visually impaired person. The LCD screen is larger. It display date and time also. Automatic off function help in power saving purpose. It is more convenient to use as well. It has 4 AAA battery in it. The accuracy factor of the pulse is $\pm 5 \%$ and for pressure is $\pm 3 \mathrm{mmHg}$. It has irregular heart beat detection in it.it cost around RS1300/-.

Beurer BC30 wrist blood pressure monitor device [53] has fully automatic blood pressure monitor in it. The memory capacity of the device is $2 \times 60$. It has WHO classification indicator in it and also arrhythmia detection. Automatic switch off, date and time display, incorrect usage message are the features in it. It also indicates when the battery is low. It cost around Rs2500/-.

Beurer Medical BM44 blood pressure monitor device [54] has fully automatic measurement. It helps in the detection of arrhythmia and it has WHO classification indicator in it. The features are larger LCD when there is error the device will give signal. It needs $4 \times 1.5 \mathrm{~V}$ AA battery. For the purpose of power saving automatic off mode is available. Battery indicator helps in detecting the low battery. It cost around Rs4400/-.

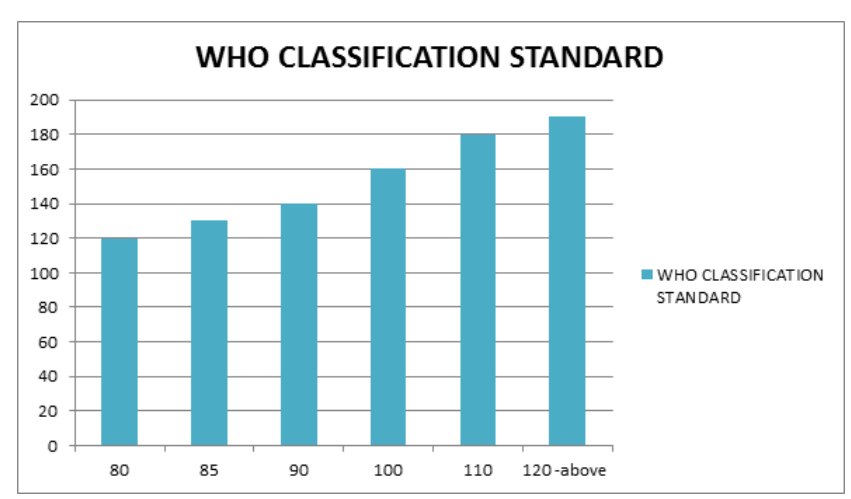

Figure 6. WHO classification standard graph (80-120 $\mathrm{mmHg})$ optimal blood pressure, (85-130 $\mathrm{mmHg}$ ) normal blood pressure, (90-140 $\mathrm{mmHg}$ ) normal systolic value, (100-160 $\mathrm{mmHg}$ ) mild hypertension, (110-180 $\mathrm{mmHg}$ ) moderate hypertension, (120- above $180 \mathrm{mmHg}$ ) severe hypertension.

I Health BP5 wireless blood pressure monitor device [55] is wireless so no need of plug or connection. It helps to check blood pressure at any places. The readings can be shared with doctor and friends. It is synchronised with iPhone application. I health mobile application is synchronized with the device and tracks systolic, diastolic pressure in mobile. It has motion sensor technology in it. The progress is displayed in dynamic chart. It has WHO indicator in it (Figure 6). The technique used in it is oscillometric method. It got certified by standard organization like European Society of Hypertension, Food and Drug Administration. It cost around Rs8000/-.

Digital Finger Oximeter, OLED pulse oximeter blood pressure monitor device [56] has unit type measuring. For the blood pressure material yon grow type is used in it. There are two OLED alarm beeps in it. For the power source, $2 \times$ AAA battery is used. And there is no oximeter for the pulse. The PR range is from $30 \mathrm{BPM}$ to $240 \mathrm{BPM}$. It cost around RS2800/-.

Karma 3AQ12P blood pressure monitor device [57] has 1HB of four types when we clicked the button. For finding the irregular heart beat PAD technology is used in it. For the accurate measurement, oscillometric method is used. The warranty period is for 1 year. The components are large led display, arm cuff, storage bag. It cost around RS 1500/-.

Dr Diaz 3.7V BP1318 Blood pressure monitor device [58] is made up of plastic material. Its main functionality is BP monitoring. For the safety purpose, the device has to be kept away from the children. As per the customer review, it is easy to use. For the power sources, 4 AA battery has to be used. Along with the device, storage kit is also provided. The instructions for placing the cuff are mention in the cuff itself so it's easy for the customer to use. It cost around RS 1300/-.

Pyle PHBPBW40BL smart blood pressure monitor [59] is a wireless device which gives instant, accurate and reliable measurement. The cuff provided in it has to be fixed in the upper arm. The cuff provided is an adjustable one so it is easy for the customer to use. Pulse rate, systolic and diastolic blood pressure, large LCD, Bluetooth with 4.0 smart technologies are 
the features of it. For the power sources, $2 \times$ AAA battery is needed. The cost of the product is RS9000/-.

Sellify generic new portable device [60] has large LCD display in it and it helps in measuring the blood pressure.it is made up of plastic material. The cuff has to be tied around the wrist region. As per the customer view, the device is not giving the accurate measurement. It has WHO indicator in it with three colours red, yellow and green. Green represent the blood pressure level is normal and yellow represent little high BP and red represent the hypertension. It cost around RS2800/-.

Niscomed PW-216 Blood pressure monitor device [61] has measuring accuracy for pressure is $\pm 3 \mathrm{mmHg}$. While measuring the blood pressure the environment can be at the temperature of 0 degree to 40 degree Celsius. Latex bulb is mainly used for the inflation process. The deflation is done by releasing the air valve. It cost around RS2500/-.

Desktop BP clock device [62] is an aneroid type blood pressure measuring device. For the easy use of the customer, there are two adjustable one is at front and other is at rear. There will be a spring present at the inflation valve. The latex tube used in it is a standard one. The components are big dial, upper arm cuff, latex pump, storage kit, instruction manual. It cost around RS1300/-.

A\&D UA-767S-W digital blood pressure monitor device [63] has indicator for measuring irregular heart beat and also if the cuff is not place in the upper arm properly there is separate indicator to detect this. Checking whether there is an error in the movement or not is done by movement indicator. It has WHO classification indicator in it. It is certified by CE and has warranty period of 5 years. It cost around RS3300/-.

Accusure $3.7 \mathrm{~V}$ device is an automatic blood pressure monitor [64] and it is made up of plastic material. It is mainly used for calculating the systolic, diastolic blood pressure. For the safety purpose, the devices have to be kept away from the children. It has warranty period for 1 year. It also display pulse per minute. It has three colour indicators in it for blood pressure measurement.

Tiny deal mini household wrist watch style digital automatic blood pressure monitor [65] measure meter tester health kit $\mathrm{HKH}-146500$ device is tied in the wrist position for measuring the blood pressure. It display systolic and diastolic blood pressure are the key features of it. It has larger LCD screen in it. The memory capacity of the device stores up to 60 measurements. For power saving purpose automatic off function is provided. It cost around RS1600/-.

AND UA-611 blood pressure monitor device [66] helps in the detection of irregular heartbeat, blood pressure monitor along with world health organisation indicator in it. The memory capacity of the device can store up to 30 readings of measurement. The cuff is mainly designed for adult. Battery indicator, systolic, diastolic pressure, pulse rate are displayed in the LCD screen. The mode of operation is one touch measurement. Arm cuff is latex and metal free one. It cost around RS1800/-.

Bang good digital device [67] is an automatic blood pressure monitor and it is tied in the wrist part. The component of the device include wrist BP monitor, instruction manual, carry box. The method used for measuring the blood pressure is oscillometric method. It has intelligent pressure sensor in it. It cost around RS9000/-.

Premium Automatic blood pressure monitor device [68] is a digital blood pressure monitor. The components of the device are arm cuff that has to be tied in the upper arm, separate manual for measuring way and sitting position.it is approved by FDA. It has memory capacity up to 60 sets of reading. It cost around RS1500/-.

Qardio Arm Wireless Blood Pressure monitor device [69] can synchronised with iOS and Android mobiles. The device is clinically validated one and it has automatic multiple user functionality in it. It mainly helps in measuring the systolic and diastolic blood pressure. It also detects irregular heartbeat. It has inflatable cuff along with the device. It cost around RS8500/-.

U.M.S. blood pressure monitor device [70] has to be tied in the upper arm. The features of the device are larger button, handy operation. The memory capacity of the device can stores up to 90 sets of readings. Room temperature display is the added feature in this device. It also detects irregular heartbeat, low battery, error in the measurement. The inflation process is done by the electric pump and deflation is an automatic process. The pressure accuracy is of $\pm 3 \mathrm{mmHg}$ and for the pulse is $\pm 5 \%$ (Table 2). When the button is not pressed for $60 \mathrm{~s}$ then the device get offed automatically. It cost around RS900/-.

Table 2. Overview of blood pressure monitor device with features.

\begin{tabular}{|c|c|c|c|c|c|c|c|}
\hline Device name & Type & & Memory capacity & Additional features & WHO classification & Battery & Cost in Rs \\
\hline I health BP 5 & Wireless & & Mobile phone storage & Results in dynamic chart & Yes & USB & 8000 \\
\hline RossmaxGB102 & Aneroid & & 3000 measurement & Blood pressure & & & \\
\hline Monitor & No & & 2 AAA battery & 1400 & & & \\
\hline RossmaxGB 101 & Aneroid & & 2000 & & & & \\
\hline Measurement & $\begin{array}{l}\text { Blood } \\
\text { monitor }\end{array}$ & pressure & No & 2 AAA battery & 1300 & & \\
\hline
\end{tabular}




\begin{tabular}{|c|c|c|c|c|c|c|}
\hline Diamond LED deluxe & Mercury & Last reading & Low-power consumption & No & $\begin{array}{l}\text { Battery or USB } \\
\text { port }\end{array}$ & 2700 \\
\hline 37-degree smart bracelet & Wearable & Mobile phone storage & Body motion monitor & & & \\
\hline Sleep tracker & NO & USB port & 2900 & & & \\
\hline JSB DBP 05 & Digital & $2 \times 90$ groups & Average calculation & Yes & 4 AA battery & 1000 \\
\hline JSB DBP04 & Digital & $2 \times 90$ groups & Average calculation & Yes & 2 AAA battery & 1000 \\
\hline Citizen $\mathrm{CH}-650$ & Digital & 60 reading & Average of 3 reading & NO & 2 AAA battery & 1800 \\
\hline MCP & Digital & $1 \times 199$ sets & Heartbeat & & & \\
\hline Pulse & No & USB & 1000 & & & \\
\hline MCP palm type & Aneroid & Last reading & Accurate results & No & Battery & 1100 \\
\hline INFI 1NF 1112A & Digital & 60 reading & Irregular heartbeat & Yes & Adopter & 1500 \\
\hline INFI FUTURA & Digital & 120 reading & Pulse & No & Adopter & 1500 \\
\hline Citizen $\mathrm{CH}-453$ & Digital & 90 reading & Body movement & & & \\
\hline Irregular heartbeat & No & Battery & 1800 & & & \\
\hline Newnik Sp501 & Aneroid & Last reading & Latex free & No & Battery & 1000 \\
\hline Covey smart bracelet & Digital cloud storage & $\begin{array}{l}\text { Synchronized with } \\
\text { phone, }\end{array}$ & & & & \\
\hline Bluetooth & No & No & USB & 2750 & & \\
\hline Beurer BC 30 & Fully automatic & $2 \times 60$ reading & Arrhythmia detection & Yes & $\begin{array}{l}4 \times 1.5 \vee \mathrm{VA} \\
\text { battery }\end{array}$ & 2500 \\
\hline Digital finger oximeter & Digital & Last stored & Alarm alert & No & $2 \times A A A$ & 2800 \\
\hline Pyle PHB PB WHOBL & Wireless & Last stored & Bluetooth & No & $2 \times \mathrm{AAA}$ & 9000 \\
\hline Sellify & Digital & Last stores & Colour indication for BP & Yes & $4 \times \mathrm{AAA}$ & 2800 \\
\hline Tiny dead & Digital and automatic & 60 reading & BP measurement & No & $2 \times$ AAA battery & 1600 \\
\hline
\end{tabular}

\section{Applications synchronized with mobile phones for blood pressure monitor device}

Blood Pressure Monitor-Family Lite application [71] was designed by Taconic system LLC mainly used by iPhone and iPad users. It has features like data visualization, medication correlation, statistical reporting, import and export email, in built reminder, easy user interface, customized data input, easy reading for the users. It helps in finding the variation of blood pressure in different arm. It helps in tracking injection type of medication. Body position tracking, glucose measurement, meal type tracking are some of the added features in it. The results are displayed to the user in the form of graphs, so it is useful for the easy understanding of the user (Figure 7 and 8). It has automatic synchronization option in it. It can be synchronized with any iOS device. The data can be exported to other devices also using the format like PDF, CSV, and plain message. Dual mode option is also available in this device with multiple user accommodation. It has warning alarm when the $\mathrm{BP}$ exits the normal pressure value.

Blood pressure-Smart blood pressure (Smart BP) BP tracker [72] was designed by Evolve Medical System. It helps in monitoring the blood pressure value and tracking the health condition regularly. This application can be synchronized with any iPhone, iPod touch and iPad devices. This application can be connected with any Microsoft health vault and apple health kit as well. The data's present in it can be shared in the PDF format. The features of this application are systolic and diastolic blood pressure monitoring, pulse rate, Body mass index (BMI), pulse pressure (PP) and mean arterial pressure (MAP). The data's can be easily stored and accessed at any time. It can synchronize with blood pressure monitor devices like A@D, Omron, Withings, Homedics, I Health, Qarido Arm. The analysis of blood pressure can be easily done by graph displayed in the application. For the security purpose, the data's are protected using touch ID protection. Intuitive colour coded data is the special feature of the application which helps in identifying the stages of the hypertension. This application is a tool to share the data's that are measured using the devices.

iCare Health Monitor [73] was developed by Beijing Jiajia Kang Kang Co. Ltd. The features of the device are blood pressure, heart rate, vision, blood lipids, vision, blood oxygen, colour blind, hearing, breath rate, lung capacity, psychological index. This is considered to be the first application for measuring these features. It has some added feature like pulse 
wave acquisition and analysis software. The accuracy factor for the heart rate is plus or minus 3. For blood pressure, the accuracy is plus or minus 12. For the blood oxygen, the accuracy is plus or minus 2. This application helps in identifying the cholesterol and the accuracy percentage is $80 \%$. The physical data are measured using Bluetooth sphygmomanometer, Bluetooth body fat scale, Bluetooth wristband, Bluetooth ECG instrument. Statistical analysis provided by means of graph. This application is mainly used for the fitness purpose and not for the medical application. This app can be upgraded but for upgrading, we have to pay the amount.

Blood pressure app: best monitor, tracker, checker [74] by Dzmitry. This application is widely used in many countries for measuring the blood pressure. The features of this application are blood pressure monitor, pulse, weight, text notes, logging drugs, regulating custom sugar-free diet. The graph displayed in this application will keep the history of blood pressure over a period of week, month and year. It also helps in tracking weight gaining and losing which adversely affect the blood pressure. The data's are kept in the calendar as it is easily available for the user to read. The data's that are present in the application can be easily shared. So it will be helpful for the doctor in giving the treatment. The data's can be backed up by the cloud using SSL protocol. So that the data's can be viewed, retrieved and synchronized. This application is a paid application in the iTunes account. The graph displays the treatment quality and progress over time, personal health notes, quick overview of history are some of the added features in it.

Blood pressure lite-BP Tracker for hypertension management [75] was designed by codulis. This application is tracking the blood pressure and also analyzing with the help of colour coded chart. The features of this application are multi user measurement, full history, and statistical display according to the time factor. It has specific tags for exercise regimes, diet change, used medications and stress level. The lite version of it can store the record value for 10 persons. The version of this application can be upgraded. This application is compatible with iOS 9.0, iPhone, iPad, iPod touch.

Blood pressure companion- BP Tracker and log [76] by Maxwell software. It mainly helps in measuring the heart rate, blood pressure and tracking weight. The results are displayed in the form of chart, histogram, and word. The main feature of this applications are the blood pressure values can be edited and added, heart rate, systolic and diastolic blood pressure, time analysis, Mean Arterial Pressure (MAP), display minimum, maximum and average number of reading, statistical data for a week, month and year, the picture data's and the snapshots can be saved as well, reminder creation, export email, passcode for the security purpose, support touch ID as passcode, Wi-Fi backup, drop box back up, 3D touch function, and apple watch version supported in it.

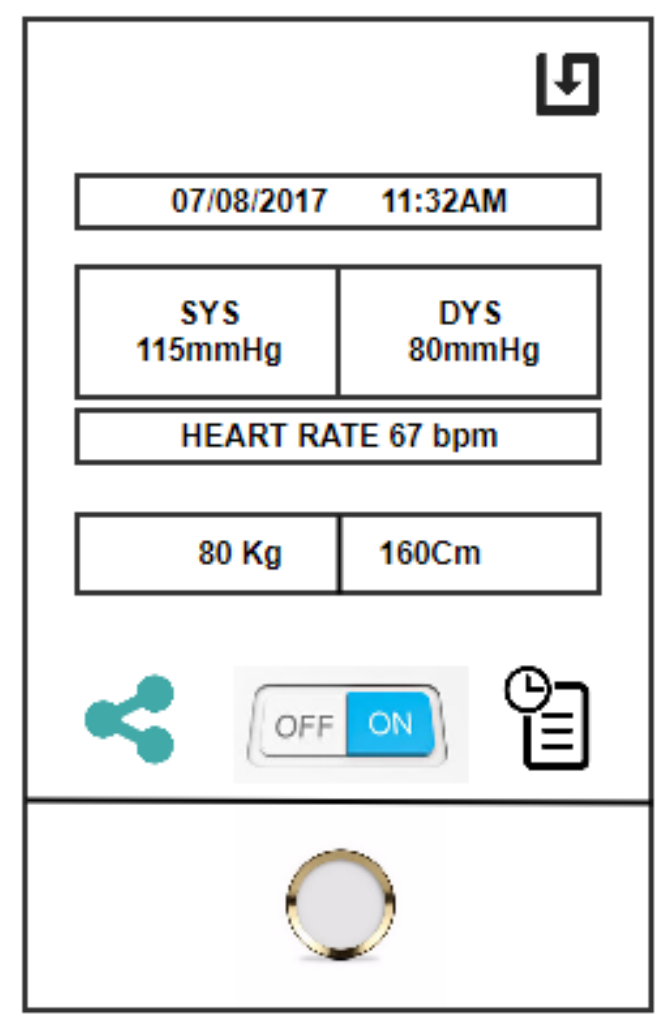

Figure 7. Blood pressure, Heart rate, Weight displayed in mobile application.

BP Wiz-Blood Pressure Log and Medication Tracker [77] by Link links LTD. It's mainly helpful in monitoring the blood pressure, heart rate, weight, chart representation for displaying the systolic, diastolic blood pressure. The key features of this application are medication log, note pad, check report per day, week, month, year basis, multiple user, custom colour range for hypertension detection, Mean Arterial Pressure (MAP), passcode protection, touch ID as passcode, import data from health application, import data from drop box, backup, restore and 3D touch function

Instant blood pressure application mainly [78] used to find the blood pressure using the smart phone and it has its own algorithm for calculating the blood pressure. For measuring blood pressure the index finger of the right hand has to be placed over the lens of the rear camera and flash. Whereas the bottom end of the phone should be placed near to the chest till the session got completed. The limitation of using instant blood pressure app ranges from $83-178 \mathrm{mmHg}$ for systolic and 58-107 $\mathrm{mmHg}$ for the diastolic. Accurate diagnosis cannot be made and when compared to the cuff its accuracy is comparatively low. 


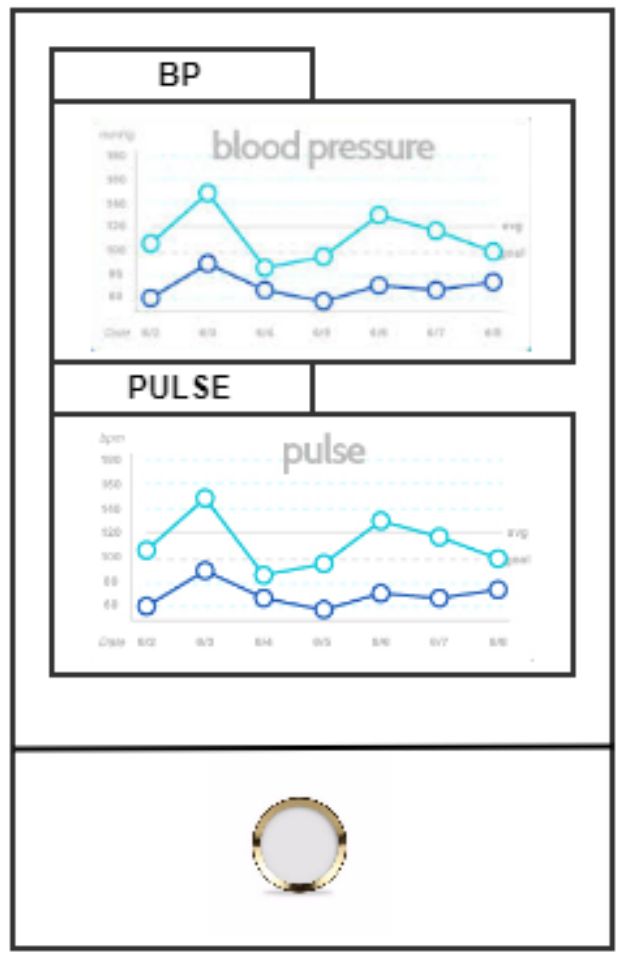

Figure 8. Graphical representations of blood pressure and pulse in mobile application.

\section{Conclusion}

The digital devices that are used for measuring the blood pressure get varied mainly by memory capacity. Even though some devices are costing high but their memory capacity can store only last three values of systolic and diastolic pressure. The accuracy factor also get varied in digital device when compared sphygmomanometer. These digital device costs high, as rural people can't make use of these devices. In future, a device with less cost, high memory capacity and high accuracy will be helpful for the rural people. Some devices can be synchronized with mobile phone which makes it extremely portable in monitoring the health condition. As hypertension is also known as silent killer disease. Predicting high blood pressure at the early stage will be helpful in improving the patient health for leading a healthy life.

\section{Acknowledgement}

This work is funded by Department of Science and Technology, Government of India and supported by VIT, Vellore, India.

\section{References}

1. http://www.amazon.in/Omron-HEM-7113-AutomaticPressure-Monitor/dp/B00CI4L2CG/ref=sr_1_1? $\mathrm{s}=$ electronics\&ie $=\mathrm{UTF} 8 \&$ qid $=150130866 \overline{6} \& \mathrm{sr}=1-1 \& \mathrm{key}$ words $=$ Automatic + Blood + Pressure + Monitor
2. http://www.amazon.in/Omron-HEM-6131-Automatic-

Pressure-Monitor/dp/B00F38B54O/ref=sr_1_1? $\mathrm{s}=$ electronics\&ie $=\mathrm{UTF} 8 \&$ qid $=1501308846 \& \mathrm{sr}=8-1 \& \mathrm{key}$ words $=$ omron +6131

3. http://www.amazon.in/Omron-HEM-7117-JPN-2Pressure-Monitor/dp/B00EH952B2/ref=sr_1_1? $\mathrm{s}=$ hpc\&ie $=$ UTF $\&$ \&id $=1501309005 \& \mathrm{sr}=1-1 \overline{1}$ keywords $=$ omron +7117

4. http://www.amazon.in/Omron-HEM-7120-AutomaticPressure-Monitor/dp/B00F38B3NW/ref=sr_1_1? $\mathrm{s}=\mathrm{hpc} \& \mathrm{ie}=\mathrm{UTF} 8 \& \mathrm{qid}=1501309359 \& \mathrm{sr}=1-1 \& \mathrm{keywords}=$ omron +7120

5. http://www.amazon.in/Omron-HEM-8712-BloodPressure-Monitor/dp/B00IAGUWUE/ref=sr_1_1? $\mathrm{s}=\mathrm{hpc} \& \mathrm{ie}=\mathrm{UTF} 8 \&$ qid $=1501309410 \& \mathrm{sr}=1-1 \& \mathrm{k}$ eywords $=$ omron +8712

6. http://www.amazon.in/Omron-HEM-7130-L-BloodPressure-Monitor/dp/B00QUWQXNU/ref=sr_1_1? $\mathrm{s}=$ hpc\&ie $=\mathrm{UTF} 8 \& \mathrm{qid}=1501309501 \& \mathrm{sr}=1-1 \&$ keywords $=$ omron+71301

7. http://www.amazon.in/Omron-HEM-7132-BloodPressure-Monitor/dp/B00FVW7T6U/ref=sr_1_1? $\mathrm{s}=$ hpc\&ie=UTF8\&qid $=1501309547 \& \mathrm{sr}=1-\overline{1} \& \mathrm{k}$ eywords $=$ omron +7132

8. http://www.amazon.in/Omron-HEM-8712-BloodPressure-Monitor/dp/B00IAGUWUE/ref=sr_1_1? $\mathrm{s}=$ hpc\&ie $=$ UTF8\&qid $=1501309594 \& \mathrm{sr}=1-\overline{1} \& \overline{\mathrm{k}}$ eywords $=$ omron +8712

9. http://www.amazon.in/Omron-HEM-7121-BloodPressure-Monitor/dp/B00F38B8GY/ref=sr_1_1? $\mathrm{s}=\mathrm{hpc} \& \mathrm{ie}=\mathrm{UTF} 8 \& \mathrm{qid}=1501309640 \& \mathrm{sr}=1-\overline{1} \& \mathrm{keywords}=$ omron +7121

10. http://www.amazon.in/Omron-HEM-7112-BloodPressure-Monitor/dp/B0074I4XJK/ref=sr_1_1? $\mathrm{s}=$ hpc\&ie $=\mathrm{UTF} 8 \&$ qid $=1501309702 \& \mathrm{sr}=1-\overline{1} \& \mathrm{keywords}=$ omron+7112

11. http://www.amazon.in/Omron-HEM-7200-JPN-1Pressure-Monitor/dp/B00F5TRP36/ref=sr_1_1? $\mathrm{s}=\mathrm{hpc} \& \mathrm{ie}=\mathrm{UTF} 8 \& \mathrm{qid}=1501309749 \& \mathrm{sr}=\overline{1}-\overline{1} \& \mathrm{keywords}=$ omron+7200

12. http://www.amazon.in/Omron-HEM-7270-PressureMonitor-Measurement/dp/B01ALRG3L6/ref=sr_1_1? $\mathrm{s}=\mathrm{hpc} \& \mathrm{ie}=\mathrm{UTF} 8 \& \mathrm{qid}=1501309824 \& \mathrm{sr}=1-1 \&$ keywords $=$ omron +7270

13. http://www.amazon.in/Omron-HEM-6121-AutomaticPressure-Monitor/dp/B00CJFDP9C/ref=sr_1_1? 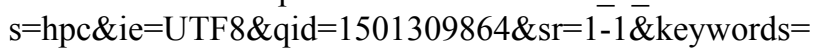 omron +6121

14. http://www.amazon.in/Omron-HEM-7117-JPN-2Pressure-Monitor/dp/B00EH952B2/ref $=$ sr_1_1? $\mathrm{s}=\mathrm{hpc} \& \mathrm{ie}=\mathrm{UTF} \& \& \mathrm{qid}=1501309916 \& \mathrm{sr}=1-1 \&$ keywords $=$ omron+7117

15. http://www.amazon.in/Omron-Wireless-Pressure-WideRange-BP786/dp/B00KW4PO82/ref=sr_1_2? $\mathrm{s}=\mathrm{hpc} \& \mathrm{ie}=\mathrm{UTF} 8 \&$ qid $=1501309956 \& \mathrm{sr}=1-2 \& \mathrm{keywords}=$ omron $+10+$ series + blood + pressure + monitor 
16. http://www.amazon.in/Omron-HEM-7200-JPN-1Pressure-Monitor/dp/B00F5TRP36/ref=sr_1_1? $\mathrm{s}=\mathrm{hpc} \& \mathrm{ie}=\mathrm{UTF} 8 \& \mathrm{qid}=1501310718 \& \mathrm{sr}=1-1 \& \mathrm{keywords}=$ omron +7200

17. http://www.amazon.in/Omron-HEM-7124-BloodPressure-Monitor/dp/B01AUH8AWW/ref=sr_1_1? $\mathrm{s}=$ hpc\&ie $=$ UTF $\&$ \& qid $=1501310796 \& \mathrm{sr}=1-1$ \&keywords $=$ omron +7124

18. http://www.amazon.in/Equinox-Digital-Pressure-MonitorEQ-BP-100/dp/B00MHSL7UU/ref=sr_1_1? $\mathrm{s}=$ hpc\&ie $=$ UTF 8 \& qid $=1501310950 \& \mathrm{sr}=1-1 \&$ keywords $=$ equinox + eq +100

19. http://www.amazon.in/Equinox-Digital-Pressure-MonitorEQ-BP-101/dp/B00BOMF62A/ref=sr_1_1? $\mathrm{s}=$ hpc\&ie $=\mathrm{UTF} 8 \&$ qid $=1501311020 \& \mathrm{sr}=1-1 \&$ keywords $=$ equinox + eq +101

20. http://www.amazon.in/Equinox-Digital-Pressure-MonitorEQ-BP-102/dp/B00BOMF86O/ref=sr_1_1? $\mathrm{s}=\mathrm{hpc} \& \mathrm{ie}=\mathrm{UTF} 8 \&$ qid $=1501311059 \& \mathrm{sr}=1-1 \&$ keywords $=$ equinox + eq +102

21. http://www.amazon.in/Equinox-Digital-Pressure-MonitorEQ-BP-102/dp/B00BOMF86O/ref=sr_1_fkmr0_4? $\mathrm{s}=$ hpc\&ie $=$ UTF 8 \&qid $=1501311230 \& \mathrm{sr}=1-4-$ fkmr0\&keywords $=$ equinox + eq $+b p+201$

22. http://www.amazon.in/Equinox-EQ-BP-54-PressureMonitor/dp/B00ESY0URC/ref=sr_1_1? 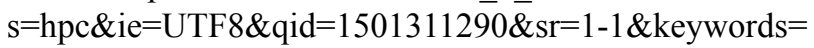 equinox $+\mathrm{eq}+\mathrm{bp}+54$

23. http://www.amazon.in/Equinox-Digital-Pressure-MonitorEQ-BP-i104/dp/B01M07JC2M/ref=sr_1_1?

$\mathrm{s}=$ hpc\&ie $=\mathrm{UTF} 8 \& \mathrm{qid}=1501311321 \& \mathrm{sr}=1-1 \&$ keywords $=$ equinox $+\mathrm{eq}+\mathrm{bp}+\mathrm{i} 104$

24. http://www.amazon.in/Healthsense-Classic-AutomaticDigital-Pressure/dp/B01E91KMB2/ref=sr_1_1? $\mathrm{s}=\mathrm{hpc} \& \mathrm{ie}=\mathrm{UTF} 8 \& \mathrm{qid}=1501311401 \& \mathrm{sr}=1-1 \&$ keywords $=$ health + sense $+b p+100$

25. http://www.amazon.in/HealthSense-Classic-BP120Automatic-Pressure/dp/B06XRCGFC5/ref=sr_1_1? $\mathrm{s}=$ hpc\&ie $=$ UTF $8 \&$ qid $=1501311444 \& \mathrm{sr}=1-1 \& \mathrm{k}$ eywords $=$ health + sense $+b p+120$

26. http://www.amazon.in/Health-Sense-Digital-PressureMonitor/dp/B0169UA75W/ref=sr_1 fkmr0 2? 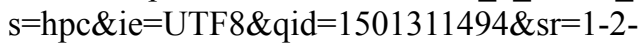 fkmr0\&keywords $=$ health + sense $+b p+300$

27. http://www.amazon.in/Health-Sense-Digital-PressureMonitor/dp/B0169UA75W/ref=sr_1_fkmr0_2? 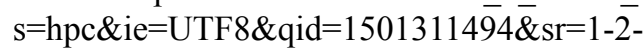 fkmr0\&keywords $=$ health + sense $+b p+300$

28. http://www.amazon.in/Dr-Trust-Automatic-HypertensionThermometer/dp/B06X9J39MK/ref=sr_1_cc_1? $\mathrm{s}=$ aps\&ie=UTF8\&qid $=1501335353 \& \mathrm{sr}=1-1-$ catcorr\&keywords $=\mathrm{dr}+$ trust + blood + pressure + monitor

29. http://www.amazon.in/Dr-Trust-Automatic-HypertensionThermometer/dp/B06X9J39MK/ref=sr_1_sc_1? $\mathrm{s}=$ hpc\&ie $=\mathrm{UTF} 8 \&$ qid $=1501339186 \& \mathrm{sr}=1-1$ spell\&keywords $=\mathrm{dr}+$ trust + usa + gold + line
30. http://www.amazon.in/Bestest-Automatic-Digital-TalkingPressure/dp/B01MSPFLZQ/ref=sr_1_1? $\mathrm{s}=$ hpc\&ie $=\mathrm{UTF} 8 \& \mathrm{qid}=1501335501 \& \mathrm{sr}=1-1 \&$ keywords $=$ bestest+bp+talk+fully

31. http://www.amazon.in/Dr-Morepen-BP3BG1-PressureMonitor/dp/B0074I5EWU/ref=sr_1_2? $\mathrm{s}=\mathrm{hpc} \& \mathrm{ie}=\mathrm{UTF} 8 \&$ qid $=1501335537 \& \mathrm{sr}=1-2 \&$ keywords $=$ $\mathrm{dr}+$ morepen $+\mathrm{bp}$

32. http://www.amazon.in/Morepen-BP-Automatic-PressureMonitor/dp/B00IW543NK/ref=sr_1_1?

$\mathrm{s}=\mathrm{hpc} \& \mathrm{ie}=\mathrm{UTF} 8 \& \mathrm{qid}=1501335537 \& \mathrm{sr}=1-1 \&$ keywords $=$ $\mathrm{dr}+$ morepen $+\mathrm{bp}$

33. http://www.amazon.in/Dr-Morepen-BP-02UA-PressureMonitor/dp/B01EJ95UK2/ref=sr_1_1? $\mathrm{s}=$ hpc\&ie $=\mathrm{UTF} 8 \&$ qid $=150133927 \overline{2} \& \mathrm{sr}=1-1 \&$ keywords $=$ $\mathrm{DR}+$ morepen $+\mathrm{bp}+3.7 \mathrm{v}$

34. http://www.amazon.in/Dr-Morepen-SPG03-AneroidPressure/dp/B0074I68GQ/ref=sr_1_1? $\mathrm{s}=\mathrm{hpc} \& \mathrm{ie}=\mathrm{UTF} 8 \&$ qid $=1501339303 \& \mathrm{sr}=1-1 \& \mathrm{keywords}=$ $\mathrm{DR}+$ morepen + spg03

35. http://www.amazon.in/Rossmax-GB102-AneroidPressure-Monitor/dp/B00BIYM9N8/ref=sr_1_1? $\mathrm{s}=\mathrm{hpc} \& \mathrm{ie}=\mathrm{UTF} 8 \& \mathrm{qid}=1501335616 \& \mathrm{sr}=1-\overline{1} \& \mathrm{keywords}=\mathrm{r}$ ossmax $+\mathrm{GB}+102$

36. http://www.amazon.in/Rossmax-AK101-Bp-Monitor/dp/ B00GV34D08/ref=sr_1_fkmr0_1? $\mathrm{s}=$ hpc\&ie=UTF $8 \&$ qid $=\overline{1}$ - $0133 \overline{5} 639 \& \mathrm{sr}=1-1-$ $\mathrm{fkmr} 0 \&$ keywords $=$ rossmax $+\mathrm{GB}+101$

37. http://www.amazon.in/Diamond-Delux-Blood-PressureMonitor/dp/B00F2FNRDA/ref=sr_1_1? $\mathrm{s}=$ hpc\&ie $=$ UTF8\&qid $=150133569 \overline{8} \dot{\mathrm{sr}}=1-1 \&$ keywords $=$ diamond + led + de

38. http:/www.amazon.in/Bluetooth-Bracelet-Wearableemotional-Smartphone/dp/B014BJJLIC/ref=sr_1_1? $\mathrm{s}=$ electronics\& $\mathrm{ie}=\mathrm{UTF} 8 \&$ qid $=1501335803 \& \mathrm{sr}=1-1 \& \mathrm{key}$ words $=37+$ degree + bluetooth + smart

39. http://www.amazon.in/JSB-DBP05-Pressure-MonitorWhite-Blue/dp/B00B6ZSZPK/ref=sr_1_1? $\mathrm{s}=$ electronics\&ie $=\mathrm{UTF} 8$ \&qid $=15013 \overline{3} 5 \overline{8} 35 \& \mathrm{sr}=8-1 \& \mathrm{key}$ words $=\mathrm{jsb}+\mathrm{dbp} 05$

40. http://www.amazon.in/JSB-DBP04-Pressure-MonitorWhite-Blue/dp/B00B6ZT07W/ref=sr_1_1? $\mathrm{s}=$ hpc\&ie $=\mathrm{UTF} 8 \&$ qid $=1501339450 \& \mathrm{sr}=1-1 \&$ keywords $=\mathrm{j}$ $\mathrm{sb}+\mathrm{dbp} 04$

41. http://www.amazon.in/Citizen-CH-650-Blood-PressureMonitor/dp/B00CCZE3F4/ref=sr_1_1? $\mathrm{s}=\mathrm{hpc} \& \mathrm{ie}=\mathrm{UTF} 8 \&$ qid $=15013358 \overline{7} \overline{\mathrm{S}} \mathrm{sr}=1-1 \&$ keywords $=$ citizen $+\mathrm{ch}+650$

42. http://www.amazon.in/Digital-Blood-Pressure-MonitorPulse/dp/B01KXX33KM/ref=sr_1_1? $\mathrm{s}=$ hpc\&ie $=$ UTF $\&$ \&id $=1501335907 \& \mathrm{sr}=1-1 \&$ keywords $=$ mcp + digital+blood + pressure

43. http://www.amazon.in/Palm-Type-AneroidSphygmomanometer-Monitor/dp/B011B91XUO/ ref $=$ sr_1_2? 
$\mathrm{s}=\mathrm{hpc} \& \mathrm{ie}=\mathrm{UTF} \& \& \mathrm{qid}=1501335969 \& \mathrm{sr}=1-2 \&$ keywords $=$ mcp+palm+blood+pressure

44. http://www.amazon.in/INF1112A-Generation-DigitalMonitor-Technology/dp/B00WLQGBG8/

ref=sr_1_fkmr0_2? $\mathrm{s}=\mathrm{hpc} \& \mathrm{ie}=\mathrm{UTF} 8 \mathrm{Q} \& \mathrm{qid}=1501336027 \& \mathrm{sr}=1-2-$ fkmr0\&keywords $=\mathrm{infi}+\mathrm{infi}+1112 \mathrm{a}+$ digital $+\mathrm{bp}$

45. http://www.amazon.in/TMB-995-Digital-MeasurementInflation-Technology/dp/B00WLQG58C/

ref=sr_1_fkmr0_1? $\mathrm{s}=\mathrm{hpc} \& \overline{\mathrm{ie}}=\mathrm{UTF} \overline{8} \& \mathrm{qid}=1501336027 \& \mathrm{sr}=1-1-$ fkmr0\&keywords $=$ infi + infi $+1112 \mathrm{a}+$ digital $+\mathrm{bp}$

46. http://www.amazon.in/s/ref=nb_sb_noss?url=search-alias $\% 3$ Dhpc\&field-keywords $=$ citizen + CH-453+bp + monitor\&rh $=\mathrm{n} \% 3 \mathrm{~A} 1350384031 \% 2 \mathrm{Ck} \% 3$ Acitizen $+\mathrm{CH}-453+\mathrm{bp}+$ monitor

47. http://www.amazon.in/Smart-Care-Mercury-PressureMonitor/dp/B01K4QR7Q4/ref=sr_1_1? $\mathrm{s}=\mathrm{hpc} \& \mathrm{ie}=\mathrm{UTF} 8 \&$ qid $=1501336197 \& \mathrm{sr}=1-1 \&$ keywords $=$ smart + care + mercury + monitor

48. http://www.amazon.in/Newnik-SP501Sphygmomanometer-Calibration-Stethoscope/dp/ B00P2G0L1K/ref=sr_1_1? $\mathrm{s}=$ hpc\&ie $=$ UTF 8 \&qid $=1501336226 \& \mathrm{sr}=1-1 \&$ keywords $=$ newnik + sp501

49. http://www.amazon.in/Smart-Care-Mercury-Dial-BPMonitor/dp/B01N7WW6JD/ref=sr_1_fkmr0_1? $\mathrm{s}=\mathrm{hpc} \& \mathrm{ie}=\mathrm{UTF} 8 \&$ qid $=1501336261 \& \mathrm{sr}=1-1-$ fkmr0\&keywords $=$ smart + care + bp + dial + type

50. http://www.amazon.in/Withings-Wireless-Blood-PressureMonitor/dp/B00H43WOAK/ref=sr_1_fkmr0_1? $\mathrm{s}=\mathrm{hpc} \& \mathrm{ie}=\mathrm{UTF} 8 \&$ qid $=150133631 \overline{5} \& \mathrm{sr}=1-1-$ fkmr0\&keywords $=$ withings + wireless $+\mathrm{bp}+$ device

51. http://www.amazon.in/iHealth-BP7-Wireless-PressureMonitor/dp/B009SPTHGQ/ref=sr_1_1? $\mathrm{s}=$ hpc\&ie $=\mathrm{UTF} 8 \&$ qid $=15013363 \overline{6} 5 \mathrm{\& sr}=1-1 \&$ keywords $=\mathrm{i}$ health + bp $7+$ wireless + device

52. http://www.amazon.in/Cooey-Smart-Bluetooth-Voicebased/dp/B06XJGBVZ2/ref=sr_1_1? $\mathrm{s}=\mathrm{hpc} \& \mathrm{ie}=\mathrm{UTF} 8 \&$ qid $=1501336404 \& \mathrm{sr}=1-1 \&$ keywords $=$ cooey+smart+bluetooth+and+voice+based+bp

53. http://www.amazon.in/Healthgenie-Monitor-digitalirregular-indicator/dp/B00P7O25HU/ref=sr_1_1? $\mathrm{s}=$ hpc\&ie $=$ UTF $\&$ \&qid $=1501336437 \& \mathrm{sr}=1-1 \&$ keywords $=$ healthgenie + digital $+b p$

54. http://www.amazon.in/Beurer-Wrist-Blood-PressureMonitor/dp/B00BLGRQHW/ref=sr 1_fkmr0_1? $\mathrm{s}=$ hpc\&ie=UTF8\&qid $=1501337281 \& \mathrm{sr}=1-1-$ fkmr0\&keywords $=$ beurer $+\mathrm{BC}+30+$ wrist + bp

55. http://www.amazon.in/Beurer-Medical-Blood-PressureMonitor/dp/B002KFZW1U/ref=sr_1_1? $\mathrm{s}=$ hpc\&ie=UTF $\&$ \&qid $=1501336514 \& \mathrm{sr}=1-1 \&$ keywords $=$ beurer+medical+BM44

56. Http://www.amazon.in/iHealth-BP5-Wireless-PressureMonitor/dp/B00ANJAKF8/ref=sr_1_sc_1? $\mathrm{s}=$ hpc\&ie $=\mathrm{UTF} 8 \&$ qid $=1501337352 \& \mathrm{sr}=1-1-$ spell\&keywords $=\mathrm{i}+$ health $+\mathrm{bp} 5+$ wireless $+\mathrm{bp}$

57. http://www.amazon.in/Digital-Oximeter-PressureMonitor-Oximetro/dp/B01M04FJM0/ref=sr_1_1? $\mathrm{s}=$ hpc\&ie=UTF $\&$ \&qid $=1501337429 \& \mathrm{sr}=1-\overline{1} \& \mathrm{k}$ eywords $=$ digital+finger+oximeter

58. http://www.amazon.in/Karma-3AQ12P-Blood-PressureMonitor/dp/B0100KGQZC/ref=sr_1_fkmr0_1? $\mathrm{s}=$ hpc\&ie=UTF8\&qid $=1501337465 \& \mathrm{sr}=1-\overline{1-}$ fkmr0\&keywords $=$ karama $+3 \mathrm{AQ} 12 \mathrm{P}$

59. http://www.amazon.in/Dr-Diaz-BP1318-Blood-PressureMonitor/dp/B01EJ95R5A/ref=sr_1_1? $\mathrm{s}=\mathrm{hpc} \& \mathrm{ie}=\mathrm{UTF} 8 \& \mathrm{qid}=1501337494 \& \mathrm{sr}=1-1 \&$ keywords $=$ $\mathrm{dr}+\mathrm{diaz}+3.7 \mathrm{~V}+\mathrm{BP}$

60. http://www.amazon.in/Pyle-BLUETOOTH-WRISRPRESSURE-PHBPBW40BK/dp/B01DMVSUQY/ ref $=\mathrm{sr} 1$ fkmr0_1? $\mathrm{s}=$ hpc \&ie $=$ UTF $\& \& q i d=1501337549 \& \mathrm{sr}=1-1-$ fkmr0\&keywords $=$ pyle + smart + bp

61. http://www.amazon.in/Sellify-Pressure-MonitorsPortable-sphygmomanometer/dp/B0744G99SH/ ref $=$ sr_1_1?

$\mathrm{s}=\mathrm{hpc} \& \mathrm{ie}=\mathrm{UTF} 8 \& \mathrm{qid}=1501337621 \& \mathrm{sr}=1-1 \&$ keywords $=$ sellify++bp

62. http://www.amazon.in/Niscomed-PW-216-BloodPressure-Monitor/dp/B00E4MI0HU/ref=sr_1_1? $\mathrm{s}=$ hpc\&ie $=$ UTF 8 \&qid $=1501337652 \& \mathrm{sr}=1-\overline{1} \&$ keywords $=$ niscomed + pw +216

63. http://www.amazon.in/UA-767SW-Home-BloodPressure-Monitor/dp/B01J5ZXCXC/ref=sr_1_fkmr0_1? $\mathrm{s}=$ hpc\&ie=UTF8\&qid=1501337716\&sr=1-1fkmr0\&keywords $=a \% 26 d+U A+767 \mathrm{~S}$

64. http://www.amazon.in/AccuSure-Automatic-BloodPressure-Monitor/dp/B01EJ95X8Q/ref=sr_1_fkmr0_1? $\mathrm{s}=$ hpc\&ie $=$ UTF $8 \& q i d=1501337775 \& \mathrm{sr}=1-1-$ fkmr0\&keywords $=$ accusure $+3.7+\mathrm{V}+$ device

65. http://www.amazon.in/Tiny-Deal-Household-AutomaticHKH-146500/dp/B00RFFUHVA/ref=sr_1_fkmr0_1? $\mathrm{s}=\mathrm{hpc} \& \mathrm{ie}=\mathrm{UTF}$ \& \& qid $=1501337871 \& \mathrm{sr}=1-1-$ fkmr0\&keywords $=$ tini + deal + mini + household + wrist +watch

66. http://www.amazon.in/AND-UA-611-UA-611-BloodPressure-Monitor/dp/B00JQ7SZLO/ref=sr_1_fkmr0_1? $\mathrm{s}=$ hpc\&ie $=\mathrm{UTF} 8 \&$ qid $=1501337972 \& \mathrm{sr}=1-1-$ fkmr0\&keywords $=$ AND $+\mathrm{UA}+611+\mathrm{bp}+$ monitor + device

67. http://www.amazon.in/Healthgenie-Monitor-Automaticirregular-indicator/dp/B00P7O285Y/ref $=$ sr_1_1? $\mathrm{s}=$ electronics\&ie $=\mathrm{UTF} 8 \&$ qid $=1501338330 \& \mathrm{sr}=8-1 \& \mathrm{key}$ words $=$ bank + digital + an + automatic + bp

68. http://www.amazon.in/Automatic-HealthyWealthyDigital-Adjustable-Sphygmomanometer-Measurement/dp/ B06XPG4BY6/ref=sr_1_1? 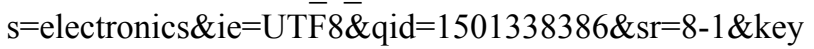 words $=$ premium + automatic + bp

69. http://www.amazon.in/QardioArm-Wireless-PressureMonitor-Android/dp/B00JM6EBHG/ref=sr_1_fkmr0_1? 
$\mathrm{s}=$ hpc\&ie $=$ UTF $8 \&$ qid $=1501338481 \& \mathrm{sr}=1-1-$

fkmr0\&keywords $=$ qardio + wireless $+\mathrm{bp}+$ monitor + device

70. http://www.amazon.in/U-M-S-BLOOD-PRESSURE-

MONITOR-UPPER-TYPE/dp/B00NIVB3EK/

ref $=$ sr_1_1?

$\mathrm{s}=\mathrm{hpc} \& \mathrm{ie}=\mathrm{UTF} 8 \& \mathrm{qid}=1501338559 \& \mathrm{sr}=1-1 \&$ keywords $=$ U.M.S+blood+pressure+monitor+device

71. https://itunes.apple.com/us/app/blood-pressure-monitorfamily-lite/id430133691? $\mathrm{mt}=8$

72. https://itunes.apple.com/us/app/blood-pressure-smartblood-pressure-smartbp-bp-tracker/id519076558? $\mathrm{mt}=8$

73. https:/itunes.apple.com/us/app/icare-health-monitor-canmeasure-blood-pressure/id1062204827?mt=8

74. https://itunes.apple.com/us/app/blood-pressure-app-bestmonitor-tracker-checker/id912831497?mt=8

75. https://itunes.apple.com/us/app/blood-pressure-lite-bptracker-for-hypertension-management/id534134435? $\mathrm{mt}=8$
76. https://itunes.apple.com/us/app/blood-pressurecompanion-bp-tracker-and-log/id458537528? $\mathrm{mt}=8$

77. https://itunes.apple.com/us/app/bp-wiz-blood-pressurelog-and-medication-tracker/id885395048? $\mathrm{mt}=8$

78. https://www.instantbloodpressure.com

\section{*Correspondence to}

Jeyanthi $\mathrm{N}$

School of Information Technology and Engineering

VIT University

Vellore

India 